\title{
What is quantum information? Information symmetry and mechanical motion
}

\author{
Vasil Penchev* \\ * Bulgarian Academy of Sciences: Institute for the Study of Societies and Knowledge: Dept. of Logical Systems and Models; vasildinev@gmail.com
}

\begin{abstract}
The concept of quantum information is introduced as both normed superposition of two orthogonal subspaces of the separable complex Hilbert space and invariance of Hamilton and Lagrange representation of any mechanical system. The base is the isomorphism of the standard introduction and the representation of a qubit to a $3 D$ unit ball, in which two points are chosen.

The separable complex Hilbert space is considered as the free variable of quantum information and any point in it (a wave function describing a state of a quantum system) as its value as the bound variable.

A qubit is equivalent to the generalization of 'bit' from the set of two equally probable alternatives to an infinite set of alternatives. Then, that Hilbert space is considered as a generalization of Peano arithmetic where any unit is substituted by a qubit and thus the set of natural number is mappable within any qubit as the complex internal structure of the unit or a different state of it. Thus, any mathematical structure being reducible to set theory is representable as a set of wave functions and a subspace of the separable complex Hilbert space, and it can be identified as the category of all categories for any functor represents an operator transforming a set (or subspace) of the separable complex Hilbert space into another. Thus, category theory is isomorphic to the Hilbertspace representation of set theory \& Peano arithmetic as above.

Given any value of quantum information, i.e. a point in the separable complex Hilbert space, it always admits two equally acceptable interpretations: the one is physical, the other is
\end{abstract}

\section{INTRODUCTION}

The history of quantum information and entanglement can be started since Neumann's Mathematische Grundlagen der Quantenmechanik (1932) [1], which (1) described mathematically rigorously the mathematical apparatus of quantum mechanics based on the separable complex Hilbert space, and (2) deduced the theorems about the absence of hidden variables in quantum mechanics on the same base. The latter implies the phenomena of entanglement in a sense ${ }^{1}$. Indeed, the separability of the interacting quantum subsystems means the availability of hidden variables, and consequently their absence according to Neumann's theorem implies entanglement as the corresponding inseparability.

\footnotetext{
${ }^{1}$ That sense is: the hidden variables in question cannot be local, so if they exist, they should be nonlocal, and this is equivalent to entanglement.
}

mathematical. The former is a wave function as the exhausted description of a certain state of a certain quantum system. The latter chooses a certain mathematical structure among a certain category. Thus there is no way to be distinguished a mathematical structure from a physical state for both are described exhaustedly as a value of quantum information. This statement in turn can be utilized to be defined quantum information by the identity of any mathematical structure to a physical state, and also vice versa. Further, that definition is equivalent to both standard definition as the normed superposition and invariance of Hamilton and Lagrange interpretation of mechanical motion introduced in the beginning of the paper.

Then, the concept of information symmetry can be involved as the symmetry between three elements or two pairs of elements: Lagrange representation and each counterpart of the pair of Hamilton representation. The sense and meaning of information symmetry may be visualized by a single (quantum) bit and its interpretation as both (privileged) reference frame and the symmetries $U(1), S U(2)$, and $S U(3)$ of the Standard model.

Key words: axiom of choice, axiom of (transfinite) induction, category theory, Hamilton representation, Hilbert space, information, information symmetry, Lagrange representation, Minkowski space, Peano arithmetic, pseudo-Riemannian space, quantum mechanics, quantum information, qubit, set theory, special \& general relativity, Standard model

The explicit formulation of the entanglement problem should refer to 1935's two papers:

Einstein, Podolsky, and Rosen's Can Quantum-Mechanical Description of Physical Reality Be Considered Complete? deduced the phenomena of entanglement from the mathematical apparatus of quantum mechanics, but considering them as reductio ad absurdum for the completeness of quantum mechanics to its incompleteness because of postulating the "elements of reality" as separable from each other. [3]

Schrödinger's Die gegenwärtige Situation in der Quantenmechanik also forecast the phenomena of entanglement calling them "verschränkten Zustände" [9]. 
However, it unlike the former paper admitted their existence in reality.

The next main stage should be connected with Bell's On the Einstein Podolsky Rosen Paradox (1964), which demonstrated that the case of entanglement can be experimentally distinguished from that of its absence formulating a sufficient (but not necessary) condition of existence: the so-called violation of Bell's inequalities. It consists in the option for the correlations between two quantities in quantum mechanics to exceed the upper limit of correlation admissible in classical mechanics ${ }^{2}$. That excess would confirm entanglement experimentally if a corresponding experiment is realized.

Kochen and Specker's paper The Problem of Hidden Variables in Quantum Mechanics (1968) [15] generalized Neumann's theorem about the absence of hidden variables in quantum mechanics as to commuting quantities as well. They elucidated that the absence of hidden variables and therefore entanglement are due to wave-particle duality and to the invariance of the discrete and continuous (smooth) motion in the final analy sis. Furthermore, the absence of hidden variables implies completeness and thus the phenomena of entanglement just for the Einstein - Podolsky - Rosen argument interpreted, however, as a confirmation rather than as reductio ad absurdum. The invariance of the discrete and continuous in tum implies the equivalence of the standard and non-standard interpretation (in the sense of Robinson's analy sis [32]) and the axiom of choice, at last.

Information (and quantum information particularly) needs fundamentally the concept of choice for both are quantities of choice measured in the units of elementary choice, correspondingly bits and qubits. Summarizing, the Kochen Specker theorem is what founds information as the real and universal substance in quantum mechanics.

The Bell's inequalities were modified into a way more convenient for experimental tests in [11], [12] and soon the results of corresponding experiments were reported [13], [14], [15]. A huge series of experiments has been realized since then including also essential modifications or complements [16], [17], [18], [19], [20], [21], [22], [23], [24], [25], [26], [27], [28].

All of them have corroborated the existence of entanglement [30], [31]. A new both fundamental and applied area of research have thrived since the 90ies of $20^{\text {th }}$ century including: quantum computer, quantum communication, and quantum cryptography.

The special attention should be paid to the so-called backdoors (or loopholes) problem. The mass of experiments confirming entanglement are indirectly and statistically admitting in principle alternative explanations without entanglement, namely "backdoors". If a certain backdoor is more or less probable as to a single experiment, that kind of explanation seems to be extremely improbable as to all corpus of those experiments. However, the reliable statistical methods

\footnotetext{
${ }^{2}$ The explanation of that kind of "super-correlations" in quantum mechanics can be ground still on the introduction of complex physical quantities unlike classical mechanics and physics where all quantities are real. Then reinterpreting the latter, "classical" case in terms of quantum mechanics, one can say that all quantities in classical mechanics and physics are postulated as independent (or "orthogonal") of each other and thus separable just according to Einstein, Podolsky, and Rosen's definition of "element of reality". [3] The "super-
}

to be unified different experiments as correlative to each other for their joint trustworthiness to be estimated are not yet elaborated.

Furthermore, all loopholes are maybe impossible to be prevented in principle [29]. For example, the conceptual base of quantum mechanics might exclude that ultimate removing any loophole for the fundamental uncertainty. [30]

Entanglement implies a new paradigm not only in quantum mechanics and physics, but also in chemistry, biology, theory of information, astronomy, cosmology, mathematics and logic, and maybe etc. It has furthermore direct reflections in metaphysics and all branches of philosophy and even in theology. Thus, it implies a fundamental turn in human knowledge.

The proofs for that should be more than convincing for the reorganization and restructuring of all human cognition would take much time, efforts, and resources. Furthermore, they would need even the alternation of generations for the change of viewpoints would be often impossible for the individuals. The thousands of present investigations would turn out to be outdated and even meaningless. For example, even maybe the biggest contemporary scientific project, GAC - CERN would turn out to be outdated needing fundamental reinterpretation.

As a result, entanglement and the corresponding theory of quantum information continue not to be officially recognized, particularly not confirmed by any Nobel prize though not refuted. They are the one alternative of the most fundamental scientific dilemma nowadays. Meanwhile, the scientific research continues in the old track though the scientific research in the new one increases. So, the state of affairs in science as to quantum information can be called ambiguous.

A qubit is usually defined as the normed superposition of two subspaces of the separable complex Hilbert space (HS) utilized in quantum mechanics. If one considers two successive "axes" of the as those two subspaces of HS, HS as well as any element or subset of it can be represent as a set of qubits.

Furthermore, one can accept two axes of one and same number belonging correspondingly to the two identical dual spaces of HS as those two subspaces able to constitute a qubit. They could not be simultaneously measured, but their superposition before measurement is postulated in the base of quantum mechanics.

At last, one can combine the latter and former interpretation of a qubit into a meta-structure isomorphic to a bit and consisting of a qubit before measurement and still two different qubits (generally, different as to each other as each to the former one).

Further, one can consider three kinds of symmetries:(1) that of each separate qubit; (2) that of each pair of qubits; (3) that of all three qubits and demonstrate that they correspond or even coincide with $U(1), S U(2)$, and $S U(3)$ accordingly. If one postulates their identity with the same symmetries of the same

correlations" in quantum mechanics are possible for the relative rotation of two quantities after they have been complex in general. The necessity of being complex in turn reflects that the quantities in quantum mechanics are probability distributions and more exactly, their characteristic functions. Then, their non-orthogonality (i.e. the availability of an arbitrary rotation between them) can be interpreted as the partial overlap of their probability distributions therefore sharing certain and ones and the same states as possible for each of them. 
name in the Standard model, a privileged triple of qubits can be determined thus and interpreted relevantly. That triple of symmetries in the general case, in which that of the Standard model is a particular case, can be call 'information symmetry' or 'quantum information symmetry'.

Meaning the usual interpretation of the two dual spaces of HS in quantum mechanics as conjugate quantities as well as the isomorphic representation of a qubit as a unit 3D ball with two points in it chosen according to a certain rule, one can identify the former qubit as the "coherent" (or "before measurement") equivalent of Lagrange representation of a point of the configurational space of the (quantum) mechanical system. Then, the latter pair of qubits would corresp ond to the Hamilton equivalent of the same.

Further, vice versa as well: one can double (or triple in a sense) any point of the configurational space of any (quantum) mechanical system, being furthermore well-ordered to a series in advance by the axiom of choice, to the qubit HS. Then, a general mathematical structure unifying classical and quantum mechanics will be obtained, after which classical and quantum mechanics will be not more than two equally acceptable interpretations of that structure: classical mechanics sees it in the way where any triple of qubits is given actually and simultaneously, and quantum mechanics correspondingly as complementary to each other, i.e. only one (though each) qubit can be given actually. Those two interpretations are different interpretations of information and its fundamental unit, a bit, in the final analysis: classical mechanics considers information as a set of alternatives not needing the axiom of choice, quantum mechanics as a choice between the same alternatives (including their set as an additional alternative).

Independently of the way of interpretation, the mathematical structure is one and the same, though. That general structure is crucial to the unification of special \& general relativity and quantum mechanics for the former shares the viewpoint of classical mechanics.

Then, the collection of all qubits representing whether any (quantum) mechanical system or all of them would be unified in a common or general space following either the approach of classical mechanics or that of quantum one. The former will result in a deformed but smooth space-time space such as the pseudo-Riemannian one of general relativity, and the latter in a "straight" but discrete space such as HS of quantum mechanics .

Meaning the general structure of HS represented by qubits, one can demonstrate not too difficultly that pseudoRiemannian space and HS are isomorphic interpretations of that qubit HS, and thus general relativity and quantum mechanics are two expressions of one and the same "state of affairs".

Meaning Skolem's concept of non-proper interpretation of any set after the axiom of choice [38] or Robinson's of nonstandard interp retation (or "nonstandard analy sis") after the slightly weaker lemma of ultrafilters [32], one can call the expression of general relativity proper or standard, and that of quantum mechanics non-proper or nonstandard. At last, one can interpret physically those mathematical conditions, under

\footnotetext{
${ }^{3}$ Any infinite set can be represented as a probabilistic distribution of all finite sets to be chosen as the correspondence of the infinite set at issue in accordance with Skolem's relativity of 'set' [38]. Then, a "wave function", i.e. an element of HS can be unambiguously
}

which the one passes into the other as well those for the reverse transformation.

The approach to generalization once unified special \& general relativity and quantum mechanics can be continued to arithmetic and set theory, and further to set theory and category theory. Here is how:

The qubit HS can be considered as a generalization of Peano arithmetic where any unit is substituted by a qubit or vice versa: one can reduce the qubit HS to Peano arithmetic if the radius of the unit 3D balls equivalent to qubits decrease to zero and they degenerate into points.

Further, one should distinguish all natural numbers according to Peano arithmetic and the set of all natural numbers according to set theory:

All natural numbers are finite according to the following simple consideration in Peano arithmetic. The number " 1 " is finite. Adding 1 to any finite number, one finite number again is their sum. Consequently, all natural numbers are finite according to the axiom of induction.

However, the set of all natural numbers is infinite according to the following in set theory. The axiom of induction is not available in it. For example, the axiom of infinity in ZFC uses a scheme isomorphic to the axiom of induction, however for justifying the opposite postulate: the postulate of infinite set. This implies that the set of all natural numbers according to set theory is infinite. In fact, any formal contradiction between Peano arithmetic and set theory does not appear since the axiom of induction is not available in the latter just as the concept of set (and thus that of infinite set) is absent in the former.

Nevertheless, the option of explicit contradiction between them exists any way for the axiom of induction and the axiom of infinity share one and the same formal scheme though interpreted in two opposite and eventually contradicting ways That option is what is realized in Gödel's incompleteness theorems (1931) [39], namely:

If one "arithmetizes” set theory e.g. as Gödel did by the so called Gödel number attachable unambiguously to any statement in set theory, this implies for the above latent contradiction between set theory and Peano arithmetic to be demonstrated explicitly:

Indeed, the arithmetized set theory is incomplete, for the infinity sets do not admit to be "arithmetized" by natural numbers being finite according to Peano arithmetic. If any way one complements Peano arithmetic with "infinity" for the arithmetized set theory to be complete, an explicit contradictory will appear: that between the finiteness of all natural numbers and the newly "infinity" as to Peano arithmetic for its unification with set theory.

One might suggest that set theory and Peano arithmetic should not be unified even implicitly, as Gödel did. However, their unification might be at the same time rather fruitful for the elucidation of the concept of infinity as dual: both "finite" in Peano arithmetic and properly "infinite" in set theory.

Particularly, Hilbert's program [40-41] for the foundation of mathematics should be restored, e.g. by interpreting " $\varepsilon$ symbol" as a probabilistic relation between finiteness and infinity therefore transferring a bridge ${ }^{3}$ also between $\mathrm{HS}$ and

juxt aposed to that probability distribution as its characteristic function. An isomorphic equivalent of entanglement can be furthermore introduced as to two or more infinite sets sharing certain finite sets as common in their corresponding probability distribution. That 
the pair of set theory and Peano arithmetic, necessary for the complete self-foundation of mathematics.

Then, the above consideration of HS as a generalization of Peano arithmetic can be complemented by it also as an equivalent of set theory at the same time.

Indeed, utilizing the axiom of choice, any set may be oneto-one mapped as a subset of all natural numbers. If that mapping is random, the set mapped is infinite, and there exist a certain non-trivial probability distribution over all finite sets (i.e. the subsets of all natural numbers) featuring it unambiguously. The characteristic function of that probability distribution will be a "wave function", i.e. an element of HS.

Thus, the $\mathrm{n}^{\text {th }}$ qubit and its value can be considered as linked to the probability for the certain infinite set at issue to be mapped into the $\mathrm{n}^{\text {th }}$ finite set.

Any qubit implies three dimensions. They may be interp reted as corresponding to the dimension of finiteness, the dimension of infinity, and the dimension of gap between them. The values of any of those dimensions are finite. All the three are independent of each other unlike the case of finiteness.

If one degenerates all sets to natural numbers and thus set theory to Peano arithmetic, all wave functions degenerate correspondingly to natural numbers. Then particularly all infinite sets turn out to be indistinguishable from each other and contradictory for all natural numbers are finite.

The completeness of mathematics cannot be proved as to a single Peano arithmetic as the so-called second Gödel incompleteness theorem demonstrates and that proof is constructive in a sense. The completeness can be proved nonconstructively as to two independent Peano arithmetics or to one single but two dimensional Peano arithmetic meaning implicitly the gap between them as the Gödel completeness theorems demonstrate. That proof is necessarily nonconstructive for it includes implicitly the gap in question.

At last, the completeness of mathematics can be proved even constructively if the gap itself is meant as a third and certain dimension as in a qubit or as in HS. Properly, the theorems about the absence of hidden variables in quantum mechanics are those or equivalent to those proofs about the completeness of mathematics.

One can approach the problem of completeness of mathematics on the base of HS otherwise:

Any "axis" of HS is interpretable as the probability for a certain numerical value to happen, i.e. be observed, measured or ascribed. Then the finite transition between two axes ${ }^{4}$ will correspond to a quantitative change within a certain quantity and therefore a certain quality, and the transfinite transition two a qualitative change, i.e. between two different qualities.

Given the intension (ideal definition) of any mathematical structure, it may be described as a finite subspace of HS, and its infinite complement to HS as its extension. Thus, any category may be associated to that subspace (or equivalently, to its complement). Any functor would be representable as an operator in HS. Those functors corresponding to self-adjoint operators would be functors within one and the same category, i.e. transforming one representative of it into some another changing only the probability for one or other property

\footnotetext{
“entanglement” cannot introduced as to two or more finite sets or an infinite set and finite sets.

${ }^{4}$ This means that the difference bet ween the ordinals of those axes is
} finite. (corresponding to a certain axis) to be ascribed to the transformed structure. Given the classical logic of predicates, only two values of probability are allowed: either " 0 " or " 1 ". This means that some properties will be excluded (" 1 "..$\rightarrow$ " 0 "), and others added (“0” $0 . \rightarrow$ “ 1 ”), and some conserved (“ 1 ” .... “ 1 ").

In other words, the self-adjoint operators can represent all operators in HS ones the above two-value logic is granted just as the "classical" quantum mechanics does.

After that has been the case (for mathematics is usually representable exhaustedly by means only of classical logic), the space of all functors turns out to be the dual space of all categories and therefore that pair will be isomorphic to the pair of the two (identical) dual spaces of HS. A new dimension of the mathematical completeness is outlined: two-valuedness or binarity. The excluded middle tends to completeness, but maybe to contradictoriness, too, if additional conventions be not involved.

Thus HS unifies both directions for the foundation of mathematics: category theory and set theory \& Peano arithmetic. Even it is able to visualize their unity in a simple and convincing one as two different viewpoints to HS: as subspaces and their transformations, and as elements and their transformations

The main conclusion is that the theory of information generalized to that of quantum information is able to unify and therefor found very extended domains of modern knowledge and contemporary cognition including those of mathematics and physics for it is able to unify the metaphysics of both ideal and material.

\footnotetext{
The paper is organized as follows:

$\checkmark$ Section 2, Quantum mechanics in terms of quantum information considers how quantum mechanics can be reinterpreted as an information theory

$\checkmark \quad$ Section 3, Summary addressing future work offers a few main directions for future work
}

\section{QUANTUM MECHANICS IN TERMS OF QUANTUM INFORMATION}

The set of all complex numbers, $\boldsymbol{C}$ is granted. Then the corresponding set of all subset of $\boldsymbol{C}$ is the separable complex Hilbert space $\mathcal{H}$.

There is one common and often met identification of $\mathcal{H}$ with the set $\mathbb{H}$ of all ordinals of $\mathcal{H}$, which rests on the identification of any set with its ordinal. However, if any ordinal is identified as a certain natural number, and all natural numbers in Peano arithmetic are finite ${ }^{5}, \mathcal{H}$ and $\mathbb{H}$ should not be equated, for $\mathcal{H}$ includes actually infinite subsets ${ }^{6}$ of $2^{C}$. Here "actually infinite subset" means "set infinite in the sense of set theory".

Furthermore, $\mathcal{H}$ is identified as the set $\mathbf{H}$ of all well-ordered sets which elements are elements of some set of $2^{C}$, i.e. in other words, the elements of $2^{C}$ considered as classes of equivalency

\footnotetext{
${ }^{5}$ This is a property implied by the axiom of induction.

${ }^{6}$ Here "actually infinite subset" means 'set infinite in the sense of set theory".
} 
in ordering are differed in ordering within any class of that ordering.

Those distinctions can be illustrated by the two basic interpretations of $\mathcal{H}:(1)$ as the vectors of $\mathrm{n}$-dimensional complex generalization of the usual 3D real Euclidean space, isomorphic to $\mathbf{H}$, and (2) as the squarely integrable functions, isomorphic to $\mathcal{H}$. The latter adds to the former unitarity (unitary invariance), which is usually interpreted as energy conservation in their application in quantum mechanics. Back seen, energy conservation is a physical equivalent of both (3) equivalence after ordering and (4) actual infinity, i.e. to (5) the concept of ordinal number in set theory.

On the contrary, once one does not involves energy conservation, e.g. generalizing it to energy-momentum conservation as in the theory of general relativity or that of entanglement, $\mathbf{H}$ rather than $\mathcal{H}$ is what should be used unlike quantum mechanics based on $\mathcal{H}$, and actual infinity avoided or at least precisely thought before utilizing.

Furthermore, (6) the relation between $\mathcal{H}$ and $\boldsymbol{H}$ can be interpreted as the 3D Euclidean space under (7) the additional condition of cyclicality (reversibility) of $\boldsymbol{H}$ conventionally identifying the first "infinite" element with the "first" element of any (trans)finite well-ordering. Indeed, the axiom of induction in Peano arithmetic does not admit infinite natural numbers ${ }^{7}$. If one needs to reconcile both finite and transfinite induction to each other, the above condition is sufficient.

It should be chosen for Poincaré's conjecture [34] proved by G. Perelman [35-37]. If that condition misses, the topological structure is equivalent to any of both almost disjunctive domains ${ }^{8}$ of Minkowski's space of special relativity ${ }^{9}$ rather than to a 4D Euclidean ball. The two domains of Minkowski space $\mathcal{M}$ can be interpreted as two opposite, "causal directions" resulting in both reversibility of the 3D Euclidean space and topological structure of the above 4D ball.

The relation between $\mathcal{H}$ and $\boldsymbol{H}$ generates any of the two areas of $\mathcal{M}$ as follows. Both unitarity of $\mathcal{H}$ and non-unitarity of $\boldsymbol{H}$ for any ordinal $n$ and any well-ordering of length $n$ are isomorphic to a 3D Euclidean sphere ${ }^{10}$ with the radius $r(n)$. All those spheres represent the area at issue.

That construction can be interpreted physically as well. Energy (E) conservation as unitarity represents the class of equivalence of any ordinal $n$. If the concept of physical force (F) is introduced as any reordering, i.e. the relation between any two elements of the above class, it can be reconciled with energy conservation (unitarity) by the quantity of distance (x) in units of elementary permutations for the reordering so that $F \cdot x=E$.

Back seen, both (6) and (7) implies Poincaré's conjecture and thus offer another way of its proof.

One can discuss the case where $\mathcal{H}$ is identified with $\boldsymbol{H}$ and what it implies. Then (8) the axiom of induction in Peano arithmetic should be replaced by transfinite induction correspondingly to (4) above, and (9) the statistical ensemble of well-orderings (as after measurement in quantum mechanics) should be equated to the set of the same elements

\footnotetext{
${ }^{7} 1$ is finite. The successor of any finite natural number is finite. Consequently, all natural numbers are finite for the axiom of induction.

${ }^{8}$ They arealmost disjunctive as share thelight cone.
}

(as the coherent state before measurement in quantum mechanics) for (3) above.

In fact, that is the real case in quantum mechanics for unitarity as energy conservation is presupposed. Then (8) implies the theorems of absence of hidden variables in quantum mechanics [1], [2], i.e. a kind of mathematical completeness interpretable as the completeness of quantum mechanics vs. Einstein, Podolsky, and Rosen's hypothesis of the incompleteness of quantum mechanics [3]:

The (8) and (9) together imply the axiom of choice. Indeed, the coherent state (the unordered set of elements) excludes any well-ordering for the impossibility of hidden variables implied by (8). However, it can be anyway well-ordered for (9). This forces the well-ordering principle ("theorem") to be involved, which in turn to the axiom of choice.

Furthermore, $\mathcal{H}$ can be represented as all sets of qubits.

A qubit is defined in quantum mechanics and information as the (10) normed superposition of two orthogonal ${ }^{11}$ subspaces of $\mathcal{H}$ :

$$
Q \stackrel{\text { def }}{=} \alpha|0\rangle+\beta|1\rangle
$$

$|0\rangle,|1\rangle$ are the two orthogonal subspaces of $\mathcal{H}$.

$\alpha, \beta \in C:|\alpha|^{2}+|\beta|^{2}=1$.

Then, (11) Q is isomorphic to a unit 3D Euclidean ball, in which two points in two orthogonal great circles ate chosen so that the one of them (the corresponding to the coefficient $\beta$ ) is on the surface of the ball.

That interpretation is obvious mathematically. It makes sense physically and philosophically for the above consideration of space as the relation of $\mathcal{H}$ and $\boldsymbol{H}$.

Now, it can be slightly reformulated and reinterpreted as the joint representability of $\mathcal{H}$ and $\boldsymbol{H}$, and thus their unifiablity in terms of quantum information.

Particularly, any theory of quantum information, including quantum mechanics as far as it is so representable, admits the coincidence of model and reality: right a fact implied by the impossibility of hidden variables in quantum mechanics for any hidden variable would mean a mismatch of model and reality.

$\boldsymbol{H}$ can be interpreted as an equivalent series of qubits for any two successive axes of $\boldsymbol{H}$ are two orthogonal subspaces of $\mathcal{H}$ :

$\left\{C_{j}\right\} \in \boldsymbol{H}$; then (12) any successive pair $\left(C_{j}, C_{j+1}\right)=Q_{j+1}$; $Q_{j+1} \in \boldsymbol{Q}$ under the following conditions:

(13) $\alpha_{j+1}=\frac{C_{j}}{\sqrt{\left(C_{j}\right)^{2}+\left(C_{j+1}\right)^{2}}} ; \beta_{j+1}=\frac{C_{j+1}}{\sqrt{\left(C_{j}\right)^{2}+\left(C_{j+1}\right)^{2}}} ;$

(14) $\alpha_{1}=0 ; \beta_{1}=\frac{C_{1}}{\left|C_{1}\right|}$;

(15) If both $C_{j}, C_{j+1}=0, \alpha_{j+1}=0, \beta_{j+1}=1$.

(14) and (15) are conventional, chosen rather arbitrarily only to be conserved a one-to-one mapping between $\boldsymbol{H}$ and $\boldsymbol{Q}$.

$\boldsymbol{Q}$ is intendedly constructed to be ambivalent to unitarity for any qubit is internally unitary, but the series of those is not. Furthermore, one can define n-bit where a qubit is 2-bit therefore transforming unitarily any non-unitary n-series of complex numbers. The essence of that construction is the double conservation between the two pairs: "within - out of" and "unitarity - nonunitarity".

\footnotetext{
${ }^{9}$ Indeed, special relativity is a causal theory, which excludes the reverse causality implied by cyclicality.

10 This means the surface of a 3D Euclidean ball.

${ }^{11}$ Any two disjunctive subspaces of $\mathcal{H}$ are orthogonal to each other.
} 
That conservation is physical and informational, in fact. The simultaneous choice between many alternatives being unitary and thus physically interpretable is equated to a series of elementary or at least more elementary choices. Then, the visible as physical inside will look like the chemical outside and vice versa. If a wholeness such as the universe is defined to contain internally its externality, this can be modeled any way consistently equating the non-unitary "chemical" and unitary "physical" representations in the framework of a relevant physical and informational conservation.

$\mathcal{H}$ can be furthermore interpreted as all possible pairs of characteristic functions of independent probability distributions and thus, of all changes of probability distributions of the state of a system, e.g. a quantum system.

Practically all probability distributions and their characteristic functions of the states of real systems are continuous and even smooth as usual. The neighboring values of probability implies the neighborhood of the states. Thus the smoothness of probability distribution implies a well-ordering and by the meditation of it, a kind of causality: the probability of the current state cannot be changed jump-like.

This is an expression of a deep mathematical dependence (or invariance) of the continuous (smooth) and discrete. The probability distribution can mediate between them as follows:

$\mathcal{H}$ can be defined as the sets of the ordinals of $\boldsymbol{H}$ where a representative among any subset of the permutations (wellorderings) of $n$ elements is chosen according a certain and thus constructive rule. That rule in the case in question is to be chosen that permutation (well-ordering), the probability distribution of which is smooth. Particularly, the homotopy of $\boldsymbol{H}$ can identified with, and thus defined as that mapping of $\boldsymbol{H}$ into $\mathcal{H}$ conserving the number of elements, i.e. the dimensionality $n$ of the vector between $\boldsymbol{H}$ and $\mathcal{H}$. If $\boldsymbol{H}$ is interpreted as the set of types on $\boldsymbol{C}$, this implies both "axiom of univalence" [4] and an (iso)morphism between the category of all categories and the pair of $\mathcal{H}$ and $\boldsymbol{H}$.

That consideration makes obvious the equivalence of the continuous (smooth) and discrete as one and the same wellordering chosen as an ordinal among all well-orderings (permutations) of the same elements and it by itself accordingly. In other words, the continuous (smooth) seems to be class of equivalence of the elements of a set (including finite as a generalization of continuity as to finite sets).

Furthermore, the same consideration can ground (3) and (9) above, i.e. the way, in which a coherent state before measurement is equivalent of the statistical ensemble of measured states in quantum mechanics. The same property can be called "invariance to choice" including the invariance to the axiom of choice particularly.

This means that the pure possibility, e.g. that of pure existence in mathematics, also interpretable as subjective probability should be equated to the objective probability of the corresponding statistical ensemble once unitarity (energy conservation) has already equated $\mathcal{H}$ and $\boldsymbol{H}$.

Indeed, the set or its ordinal can be attributed to the elements of $\mathcal{H}$ and the statistical mix of all elements of $\boldsymbol{H}$ corresponding to a given element of $\mathcal{H}$. Any measurement ascribes randomly a certain element of the corresponding subset of $\boldsymbol{H}$ to any given element of $\mathcal{H}$. Thus measurement is not unitary, e.g. a collapse of wave function.
Then, $\mathcal{H}$ and $\boldsymbol{H}$ can be interpreted as two identical but complementary dual spaces of the separable complex Hilbert space. Initarity means right their identity, and the non-unitarity of measurement representing a random choice means their complementarity.

That "invariance to choice" can ground both so-called Born probabilistic [5] and Everett (\& Wheeler) "many-worlds" interpretations of quantum mechanics [6], [7], [8]. The former means the probability for a state to be measured or a "world" to take place, and the former complement that consideration by the fact that all elements constituting the statistical ensemble can be consistently accepted as actually existing.

One can emphasize that the Born interpretation ascribes a physical meaning of the one component (namely the square of the module as probability) of any element of the field of complex numbers underlying both $\mathcal{H}$ and $\boldsymbol{H}$. After that, the physical meaning of the other component, the phase is even much more interesting. It should correspond to initarity, and then, it seems to be redundant, i.e. the field of real numbers would be sufficient, on the one hand, but furthermore, to time, well-ordering, and choice implied by it. In other words, just the phase is what is both physical and mathematical "carrier" and "atom" of the invariance of choice featuring the separable complex Hilbert space.

\section{DIRECTIONS ADDRESING FUTURE WORK}

Those are:

(1) Hamilton and Lagrange interpretations unified in quantum information should explain how the concept of (quantum) bit unifies both ways for mechanic to be interpreted.

(2) Information as the quantity of choice(s) discusses why information is the quantity of choices in the final analysis.

(3) HS as a generalization of Peano arithmetic deduces how the separable complex Hilbert space can be seen as a generalization of Peano arithmetic and the conclusion about the foundation of mathematics.

(4) Models of set and category theory in HS elucidates how HS can unify set theory and category theory.

(5) Identifying physics and mathematics as interpretations of quantum information reveals why a state in quantum mechanics and a mathematical structure in mathematics are isomorphic to each other as two equally admissible interpretations of quantum information

(6) Information symmetry introduces the concept of information symmetry on the base of the equivalence of Hamilton and Lagrange interpretations

(7) Information symmetry visualised by impressing examples exemplifies it by the symmetries of three qubits and their interpretation as a privileged reference frame

(8) Metaphysical and philosophical interpretations would discuss (quantum) information as the general substance of all mental and material phenomena. 


\section{REFERENCES}

[1] Neuman, J. von. MathematischeGrundlagen der Quantenmechanik. Berlin: Springer, pp. 167-173(1932).

[2] Kochen, S., Specker, E. The Problem of Hidden Variables in Quantum Mechanics. Journal of Mathematics and Mechanics 17(1): 59-87 (1968).

[3] Einstein, A., Podolsky, B., Rosen, N. Can Quantum-Mechanical Description of Physical Reality Be Considered Complete? Physical Review 47(10): 777-780.

[4] (Institute for Advanced Study, Princeton, NJ, Univalent Foundations Program) Homotopy type theory: univalent foundations of mathematics. Princeton, NJ: Lulu Press, Univalent Foundations Program (2013)

[5] Max Born - Nobel Lecture: The Statistical Interpretations of Quantum Mechanics". Nobelprize.org. Nobel Media AB 2014. Web. 13 Jul 2016.

<http://www.nobelprize.org/nobel_prizes/physics/laureates/1954/bom -lecture.html>

[6] Everett III, H. "Relative State" Formulation of Quantum Mechanics Reviews of Modern Physics 29(3): 454-462 (1957).

[7] DeWitt, B. S., Graham, N. (eds.) The many-worlds interpretation of quantum mechanics: a fundamental exposition. Princeton, NJ: University Press (1973)

[8] Wheeler, J. A., Zurek, W. H (eds.). Quantum theory and measurement. Princeton, N.J.: Princeton University Press, 1983.

[9] Schrödinger, E. Die gegenwärtige Situation in der Quantenmechanik. Naturwissenschaften 23(48): 807-812; (49): 823-828; (50): 844-849 (19350.

[10] Bell, J. On the Einstein Podolsky Rosen Paradox. Physics (New York) 1(3): 195-200 (1964).

[11] Clauser, J.F; Horne, M.A.; Shimony, A.; Holt, R.A, Proposed experiment to test local hidden-variable theories, Physical Review. Letters 23 (15): 880-884(1969).

[12] Clauser, J. F.; Horne, M. A. Experimental consequences of objective local theories. Physical Review D 10 (2): 526-535 (1974).

[13] Freedman, S.J; Clauser, J.F. Experimental test of local hiddenvariable theories. Physical Review Letters 28(938): 938-941 (1972).

[14] Aspect, A., Grangier, P.; Roger, G., Experimental Tests of Realistic Local Theories via Bell's Theorem. Physical Review Letters 47(7): 460-463 (1981)

[15] Aspect, A., Dalibard, J; Roger, G. Experimental Test of Bell's Inequalities Using Time-Varying Analyzers. Physical Review Letters 49(25): 1804-1807 (1982).

[16] Tittel, W. et al. Experimental demonstration of quantumcorrelations over more than 10 kilometers. Physical Review A 57(5): 3229-3232 (1998).

[17] Tittel, W. et al. Violation of Bell inequalities by photons more than 10 km apart. Physical Review Letters 81(17): 3563-3566 (1998).

[18] Weihs, G. et al. Violation of Bell's inequality under strict Einstein locality conditions. Physical Review Letters 81(23): 5039-5043 (1998).

[19] Pan, Jian-Wei et al. Experimental test of quantum nonlocality in three-photon GHZ entanglement. Nature 403 (6769): 515-519 (2000).

[20] Rowe, M.A. et al. Experimental violation of a Bell's inequality with efficient detection. Nature 409 (6822): 791-794 (2001).

[21] Gröblacher, S. et al. An experimental test of non-local realism. Nature 446(7138): 871-875 (2006).

[22] Salart, D et al. Spacelike Separation in a Bell Test Assuming Gravitationally Induced Collapses. Physical Review Letters 100 (22): 220404-4(2008).

[23] Ansmann, M. et al. Violation of Bell's inequality in Josephson phase qubits. Nature 461(7263): 504-506: (2009).

[24] Giust ina, M. et al. Bell violation using ent angled photons withou the fair-sampling assumption. Nature 497 (7448): 227-230 (2013).
[25] Larsson, Jan-Åke et al. Bell violation with entangled photons, free of the coincidence-time loophole. Physical Review A 90(3): 032107-7 (2014)

[26] Christensen, B.G. et al. Detection-Loophole-Free Test of Quantum Nonlocality, and Applications. Physical Review Letters 111(13): 130406-5. (2013)

[27] Giustina, M.et al. A significant-loophole-free test of Bell's theorem with entangled photons. Physical Review Letters 115(25): 250401-7(2015)

[28] Shalm, L. K. et al. A strong loophole-free test of local realism. Physical Review Letters 115(25): 250402-10 (2015).

[29] Brunner, N. Bell nonlocality. Review of Modern Physics 86(2): 419-478(2014).

[30] Santos, E. (2005), Bell's theorem and the experiments: Increasing empirical support to local realism. Studies In History and Philosophy of Modern Physics 36(3): 544-565(2005)

[31] Wiseman, H. Quantum physics: Death by experiment for local realism. Nature 526(7575): 649-650(2015).

[32] Robinson, A. Non-standard analysis. Amsterdam: North-Holland Publishing. Company (1966).

[33] Noether, E. Invariante Variationsprobleme. Nachrichten von der Gesellschaft der Wissenschaften zu Göttingen, MathematischPhysikalische Klasse - 1918, pp. 235-257 (1918).

[34] Poincaré, H. Cinquième complément à l'analysis situs. Rendiconti del Circolo Matematico di Palermo. 1. Memorie e communicazioni 18(1): 45-110(1904).

[35] Perelman, G. The entropy formula for the Ricci flow and its geometric applications. arXiv:math.DG/0211159v1 [math.DG] (2002).

[36] Perelman, G. Ricci flow with surgery on three-manifolds. arXiv:math.DG/0303109v1 [math.DG] (2003).

[37] Perelman, G. Finite extinction time for the solutions to the Ricci on certain three-manifolds. arXiv:math.DG/0307245v1 [math.DG] (2003).

[38] Skolem, T. Einige Bemerkungen zur axiomatischen Begründung der Mengenlehre. Matematikerkongressen i Helsingrofs den 4-7 Juli 1922, Den femte skandinaviska matematikerkongressen, Redogörelse, Helsinki: Akademiska-Bokhandeln, 217-232 (1923).

[39] Gödel, K. Über formal unentscheidbare Sätze der Principia mathematica und verwandter Systeme I. Monatshefte der Mathematik und Physik, 38(1): 173-198(1931).

[40] Hilbert, D. Die Grundlagen der Mathematik. Abhandlungen aus dem Seminar der Hamburgischen Universität, 6: 65-85(1928).

[41] Hilbert, D, Bernays, P. Grundlagen der Mathematik (Vol. 2). Berlin, New York: Springer (1939). 